\title{
Impacts of top-end vessel sway on vortex-induced vibration of the submarine riser for a floating platform in deep water
}

\author{
Weimin Chen ${ }^{\mathrm{a}, *}$, Min Li ${ }^{\mathrm{b}}$, Zhongqin Zheng ${ }^{\mathrm{a}}$, Shuangxi Guo ${ }^{\mathrm{a}}$, Kuan Gan ${ }^{\mathrm{b}}$ \\ ${ }^{a}$ Key Laboratory for Mechanics in Fluid Solid Coupling System, Institute of Mechanics, Chinese Academy of Sciences, Beijing 100190, China \\ ${ }^{\mathrm{b}}$ School of Aeronautics Sciences and Engineering, Beijing University of Aeronautics and Astronautics, Beijing 100191, China
}

\section{A R T I C L E I N F O}

\section{Article history:}

Received 11 October 2013

Accepted 3 March 2015

Available online 19 March 2015

\section{Keywords:}

Sway

Fluid solid coupling

Riser

Vortex-induced vibration

\begin{abstract}
A B S T R A C T
The dynamic coupling between moving top-end vessel and submarine riser becomes more remarkable for floating platform in deep water due to its larger amplitude of top-end motion, compared to fixed platform in shallow water. The impacts of top-end motion on the riser undergoing vortex-induced vibration (VIV) are explored in this study. A coupled hydrodynamic force approach, involving the vortex-induced lift force along with the fluid drag force, is developed, which takes into account the interaction between fluid dynamic force and instantaneous riser motion. Then the dynamic behaviors of the riser suffering both top-end motion and VIV are examined by means of finite element simulations. The effects of the amplitude and frequency of topend vessel sway on riser's VIV are studied. During the riser's dynamic responses, an interesting phenomenon, called nonlinear response amplification, is observed, which demonstrates that top-end motion may be amplified as the motion propagates along riser length. Our numerical results show that the riser's displacement becomes several times larger than that of the case without top-end motion. Moreover, the nonlinear amplification gets more pronounced as the number of mode order drops, but the amplification factor just slightly changes with the increase of sway amplitude.
\end{abstract}

(c) 2015 Elsevier Ltd. All rights reserved.

\section{Introduction}

Submarine riser of deep water platform has been becoming longer and longer as the oil and gas exploration extends toward deeper ocean. Since the aspect ratio (the ratio of structural length to diameter) of deep water riser, connecting top vessel and sea bed, is getting larger, i.e. to a magnitude order of $10^{2}$ or $10^{3}$, structural modal frequencies are often low and dense. And in practice, the fluid field characters, including speed value and direction of sea current or wave, are no longer uniformly distributed along riser length. Therefore some complicated phenomena, e.g. multi-mode vibration and wider-band random vibration, of the vortex-induced vibration of long flexible risers are frequently observed (Chen et al., 2006; Gu et al., 2012; Heurtier et al., 2001; Lou et al., 2010; Stansberg et al., 2002; Tahara and Kim, 2003). On the other hand, compared to fixed platform in shallow water, floating platform in deep water has more significant motion amplitude, and the coupling between top-end vessel and submarine riser appears to be more remarkable. Moreover, new issues such as additional lock-in region, parameter excitation and nonlinear amplification, due to coupling effects are introduced (Garrett, 2005; Lei et al., 2010; Wang and Ling, 1998). It is found that the tension fluctuation due to top-end

\footnotetext{
*Corresponding author. Tel.: +8610 82543891; fax: +8610 82338527 .

E-mail address: wmchen@imech.ac.cn (W. Chen).
}

heave may introduce new riser's VIV involving higher-order modes and larger dynamic responses, e.g. riser's displacement and shear stress are respectively $10 \%$ and $20-100 \%$ larger than the case without vessel motion (Wang and Ling, 1998).

Among those research on the dynamic coupling between top-end vessel and marine structures (like riser, tension leg and mooring lines), most of them focus on dynamic response of top-end vessels rather than submarine structures. Generally, the analysis methods concerning interactions of top-end vessel and marine structures can be classified into two kinds: the quasi-static method (Ormberg et al., 1997; Kim et al., 2001; Spanos et al., 2005; Wichers et al., 2001; Xu et al., 2009) and the coupled method (Bosman and Hooker, 1999; Chen et al., 2006; Gu et al., 2012; Li et al., 2010; Tahara and Kim, 2003). In the quasi-static method, riser is simplified as a spring with lumped mass. In that case, only the static restoring force, due to wet weight and (or) elastic stiffness of risers, is taken into account. For example, Spanos et al. (2005) studied the influence of riser stiffness on the overall dynamic response of a SPAR platform by using a simplified model, in which the top-end vessel mass was concentrated at the gravity center and a horizontal spring was used to simulate the interaction between riser and vessel. $\mathrm{Xu}$ et al. (2009) considered the structural nonlinear property of a tension leg and presented the comparison between two different tension leg models, i.e. the nonlinear beam and the massless spring model. He pointed out that the dynamic responses of tension leg platform based on different models are significantly different. Ormberg et al. 
(1997), Kim et al. (2001) and Wichers et al. (2001) investigated the interactions of floating top-end and riser (or mooring system) of deepwater platforms so as to compare the coupled approach in time-domain and the quasi-static approach. Their results show that the capability of mooring system bearing external loads may be underestimated by using the quasi-static approach.

Actually, the coupled methods might be further classified into fully-coupled method and weakly-coupled method. In fully-coupled method, dynamic response of a system involving top-end and submarine structures is analyzed by considering at least two dynamic interactions, one is the interaction between environmental loads and floating vessel, another one is the interaction between floating vessel and submarine structures. In weakly-coupled method, one (or two) of the above interactions is simplified or even neglected. In most of the coupled methods, fully or weakly coupled, submarine riser and hydrodynamic force exerted by ocean current or wave are mostly simplified (Lee and Wang, 2000; Li et al., 2010; Tahara and Kim, 2008), e.g. the Morison formula is employed to model hydrodynamic force. The primary concern of the existing research lies in riser's dynamic responses rather than riser's VIV. Lee and Wang (2000) used a linear tensional string to simulate tension leg and analyzed the leg dynamic response as the top-end vessel experiencing periodical surge. His results showed that there is a leg vibration mode similar with the platform while the vibration amplitude changes with wave period. Tahara and Kim (2008) employed the empirical formula of the Young's modulus suggested by Bosman and Hooker (1999) to examine the mooring system response of a SPAR undergoing heave. By comparing his result with that of a linearly elastic mooring system, he found a remarkable difference between the two results.

In addition, it is worthwhile to point out that the dynamic coupling mechanisms, between top vessel and submarine riser, due to different kinds of vessel motions are essentially different. Taking vessel heave as an example, it introduces a fluctuating tension of riser, which presents a periodically-varying structural property. Thus, the consequence is a parameter excitation of riser. Whereas, if taking horizontal motions, i.e. sway or surge of vessel, under consideration, the transverse vibration of top-end will propagate along riser length. This transverse vibration may directly interact with riser's VIV. Moreover, the vibration might be amplified during its propagation along riser length. This effect of top-end sway, in essence, introduces a quite different issue from parameter excitation due to top-end heave. Here, only the dynamic interaction between top-end vessel sway and riser's VIV is addressed. And, we consider the weaklycoupling issue, since the fully-coupled approach is costlier than the weakly-coupled approach in terms of computer source and time.

First, we develop a hydrodynamic approach to model the vortexinduced lift force which essentially depends on structural motion. Then the dynamic response of the riser simultaneously suffering topend sway and VIV is examined by means of finite element simulations during which the top-end is assumed to be in a, for representation and simplicity, periodically sinusoidal motion. Effects of top-end sway amplitude and frequency on riser's response displacement as well as vibration propagation are examined so as to have a deeper insight into impacts of top-end vessel sway on riser's VIV.

\section{Numerical model of dynamic response analysis for integrated system}

\subsection{Structure model}

The system including the top-vessel and riser is shown in Fig. 1. In Fig. $1 \mathrm{a}$, the origin point of the coordinate system is located at the bottom end of the riser (fixed to the sea bed). The flow $U$ directs along the axis $y$. The sway motion of top vessel, $b(t)=B e^{-i \omega_{0} t}$, is along the axis $x$, where $B$ and $\omega_{0}$ are respectively the amplitude and
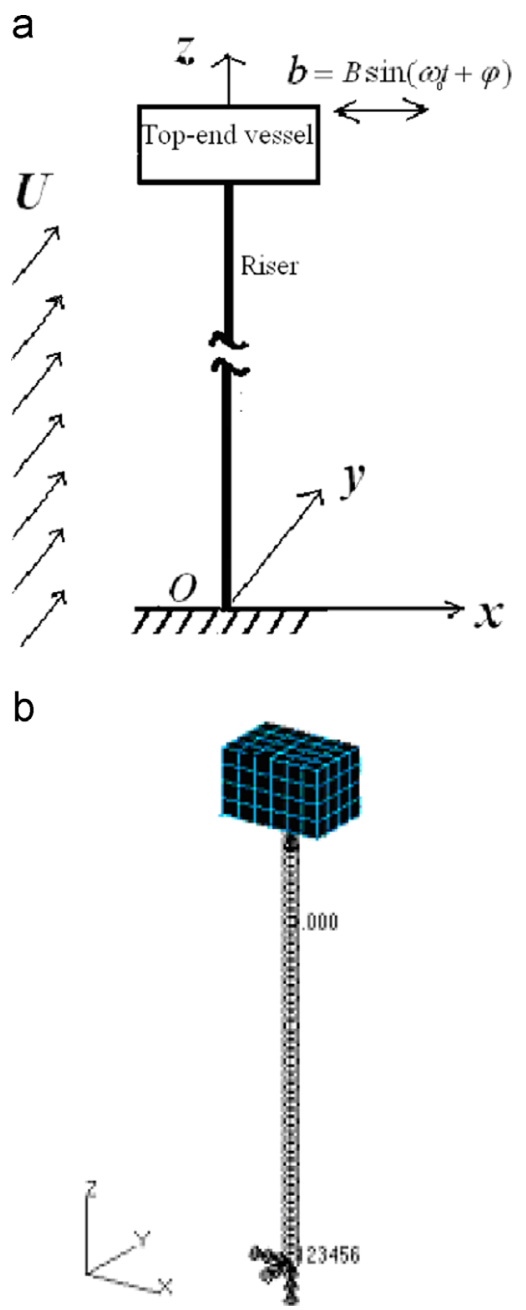

Fig. 1. The platform-riser system sketch: (a) the platform-riser system sketch and (b) the finite element model of platform-riser system.

frequency of the sway motion. In the finite element model (shown in Fig. 1b), the vessel and the riser respectively consist of 3D cubic solid and 1D Euler beam elements. The rotational motions around the axis $x, y$, and $z$ of all those grids of the top-end body are constrained during the numerical simulations so as to avoid a probable singularity introduced by the extremely large mass of the top-end. Additionally, the multi-point constraint (MPC) is used at the joint grid connecting the top-end vessel and the riser, where different elements meet together, so that the constraints can be applied effectively and smoothly upon different degrees of freedom.

The governing equation of the riser dynamics can be written as

$E I \frac{\partial^{4} x(z, t)}{\partial z^{4}}-T \frac{\partial^{2} x(z, t)}{\partial z^{2}}+c \frac{\partial x(z, t)}{\partial t}+m \frac{\partial^{2} x(z, t)}{\partial t^{2}}=F(z, t)$

where $E I$ is the bending stiffness, $T$ is the top tension, $c$ is the structural damping, and $m$ is the structural mass per unit length. $F(z)$ is the hydrodynamic force, of which the expression model will be presented in Section 2.2. The boundary conditions at two ends of the riser are

$$
\begin{gathered}
x(0, t)=0 \\
\partial^{2} x(0, t) / \partial z^{2}=0
\end{gathered} \text { and } \begin{gathered}
x(L, t)=b(t) \\
\partial^{2} x(L, t) / \partial z^{2}=0
\end{gathered}
$$

\subsection{Hydrodynamic force model}

The fluid wake field of a riser undergoing VIV is too complicated to directly get a theoretical solution because of the uncertainties like 
turbulence, separation and boundary layer. In this study, a semiempirical expression of lift force in wake field is developed based on the experimental data along with the physical mechanism governing the unique traits of VIV. The right side of Eq. (1), the hydrodynamic force $F(z)$, consists of two parts, i.e. the vortex-induced lift force $F_{L}$ and the fluid drag force $F_{D}$. The fluid drag force $F_{D}$ can be expressed by the Morison equation as (Sarpkaya, 2004)

$F_{D}=\frac{1}{2} C_{D} \rho_{f} D(U-\dot{x})|U-\dot{x}|+\frac{1}{4} C_{a} \pi D^{2} \rho_{f}(\dot{U}-\ddot{x})+\frac{1}{4} \pi D^{2} \rho_{f} \dot{U}$

where $\rho_{f}$ and $U$ are the fluid density and velocity respectively. $D$ is the riser outer diameter. $C_{D}$ and $C_{a}$ are the coefficients of added mass and drag forces respectively, of which the values are $C_{a}=1.0$ and $C_{D}=1.1$ for a flexible riser with large aspect ratio (Sarpkaya, 2004; Chen et al., 2012).

When it comes to the vortex-induced lift force $F_{L}$, its expression is more complicated because VIV has always been a challenging issue concerning the interaction between fluid and structural dynamics. VIV exhibits some interesting, and even unexplained until now, traits like self-excitation, self-limitation of response amplitude, a variety of vortex-shedding modes, multi-mode or wide-band random vibration. Originally, one of ways to express the vortex-induced lift force per unit length of a riser is, somewhat similar with the Morison equation, as

$F_{L}=\frac{1}{2} \rho_{f} U^{2} C_{L} D$

where the lift coefficient $C_{L}$ is a constant value ranging usually from 0.8 to 1.2 (Xu et al., 2009; Wang and Ling, 1998).

With recently increasing experimental observations, along with plenty of CFD simulations, it is noted that it would be more reasonable and accurate if the coupling between structural and fluid dynamics is included in hydrodynamic force model (Gopalkrishnan, 1993; Vandiver, 2002; Sarpkaya, 2004; Chen et al., 2012). Sarpkaya(2004) experimentally measured the Fourier average of hydrodynamic force

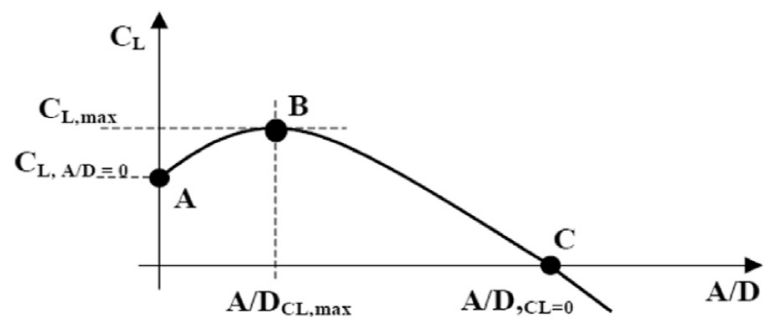

Fig. 2. The lift coefficient curve. over many cycles of vibration. He decomposed the lift force into two parts, the drag part and the inertia part, which are respectively related to velocity and acceleration of body motion. He pointed out that for practical range of Reynolds numbers, the nonlinear expression in terms of structural motion is able to capture the hydrodynamic feature better than the linear expression. Gopalkrishnan (1993) and Govardhan and Williamson (2004) systematically implemented many VIV experiments and presented the lift coefficient plots versus structural motion. Vandiver (2002) suggested that a piecewise parabola function of structural amplitude could be used for industrial model of lift force to calculate riser's displacement by using the wake oscillator model. Based on these fruitful and inspiring results, we think that the lift coefficient, $C_{L}$, should depend on structure motion rather than a merely constant value.

Here, a third-order polynomial of the structure displacement (shown in Fig. 2) is proposed to model the lift coefficient so as to take account of the nonlinear interaction between structural and fluid dynamics as follows:

$$
\begin{aligned}
F_{L}(z) & =\frac{1}{2} \rho_{f} U^{2} D\left(C_{L 0}+C_{1} \bar{x}_{0}(z)+C_{2} \bar{x}_{0}^{2}(z)+C_{3} \bar{x}_{0}^{3}(z)\right) \\
& =p_{f} C_{L}\left(\bar{x}_{0}(z)\right)
\end{aligned}
$$

where $p_{f}=1 / 2 \rho U^{2}$ Dand $\bar{x}_{0}(z)=A(z) / D$ is the non-dimensional amplitude of structural displacement. $C_{L}(\bar{x}(z))=C_{L 0} \sin (\omega t)+$ $C_{1} \bar{x}(z)+C_{2} \bar{x}^{2}(z)+C_{3} \bar{x}^{3}(z)$ is the lift coefficient. The values of coefficients $C_{L 0}, C_{1}, C_{2}$ and $C_{3}$ can be experimentally determined. Taking Gopalkrishnan's experimental plot of lift coefficient as an example (Gopalkrishnan, 1993; as shown in Fig. 3), we can obtain the horizontal coordinate value of point $C, A / D \mid C_{L}=0$, in Fig. 2 by corresponding it to a point of curve 1 in Fig. 3 . And, we can obtain the vertical coordinate value of point $A,\left.C_{L}\right|_{A / D=0}$, in Fig. 2 by corresponding it to a point of curve 4 in Fig. 3. In addition, with the assumption of a zero value of the slope at point $B$ in Fig. 2, we can get the values of coefficients $C_{L 0}, C_{1}, C_{2}$ and $C_{3}$ in Eq. (4) by solving an equation group (Chen et al., 2012).

Observing Eq. (4), we may say that this equation is capable of, to some extent, capturing some natural features of VIV. (1) The feature of self-excitation. As we know, an action of excitation will happen to the system as reduced velocity falls into lock-in range. In Eq. (4), the excitation is represented by the first two terms, i.e., the constant excitation term $C_{L O} p_{f}$ and $C_{1} p_{f} \bar{x}_{0}(z)$ which increases as vibration amplitude $\bar{x}_{0}$ increases ( $C_{1}$ is required to be positive). (2) The feature of self-limitation. One of the unique traits of VIV is that structural amplitude will never rise infinitely but start to drop when its value reaches to a certain number, such as $\bar{x}_{\max }=1.5$ or 2.0. It indicates that the excitation of resonance becomes weaker while the damping is

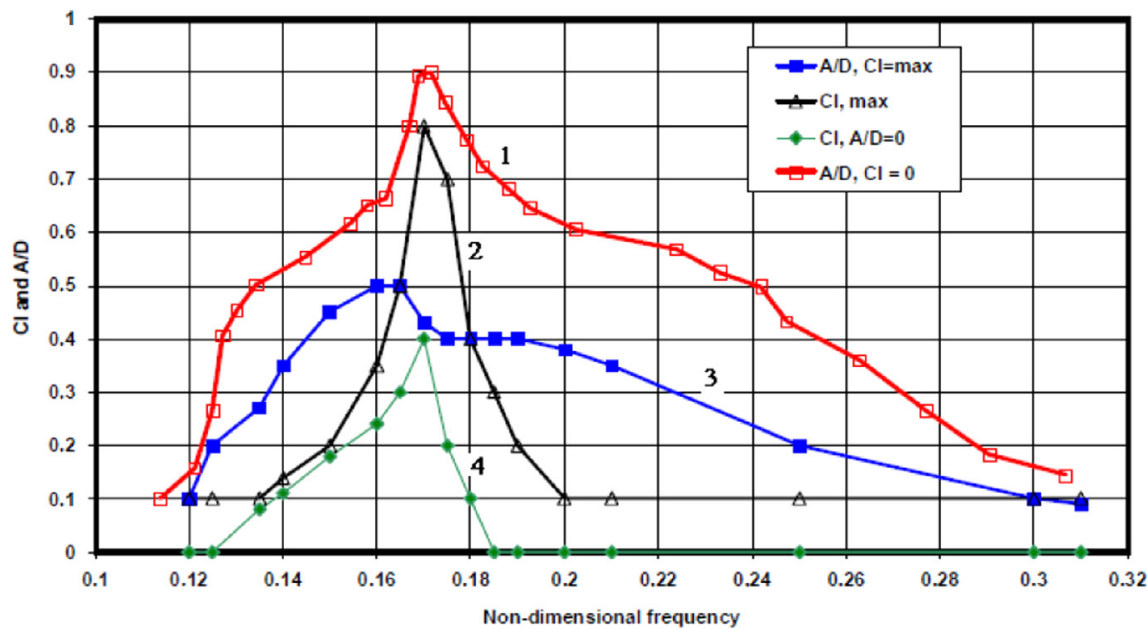

Fig. 3. Plots lift coefficient, as well as amplitude, versus nondimensional frequency (Gopalkrishnan, 1993). 
getting stronger. This feature, called self-limitation, is represented by the nonlinear terms of $\bar{x}(z)$ in Eq. (4), i.e. $C_{2} \rho_{f} \bar{x}_{0}^{2}(z)$ or $C_{3} \rho_{f} \bar{x}_{0}^{3}(z)$ (at least one of the two coefficients $C_{2}$ and $C_{3}$ is negative). (3) Axiallyvarying distribution of lift force along riser length. For the case of a rigid cylinder, the vortex-induced lift force uniformly distributes along riser length, and consequently the coherent length is equal to the length of the riser. But for the case of a flexible slender riser, the coherence may reduce due to the non-uniform distributions of lift force as well as structural motion. In our model, the lift force is certainly non-uniform because of the axially-varying vibration amplitude. Therefore, this model automatically captures the natural span coherence of flexible riser undergoing VIV.

We also numerically explored the effect of each term in Eq. (4), i.e. the constant term $C_{L 0} p_{f}$, the linear term $C_{1} p_{f} \bar{x}_{0}(z)$ and the nonlinear terms $C_{2} \rho_{f} \bar{x}_{0}^{2}(z)$ and $C_{3} \rho_{f} \bar{x}_{0}^{3}(z)$, on the structure responses. Generally speaking, the constant and linear terms behave as excitation while the nonlinear terms behave as damping to system vibration. So, based on the presented model, the lift force will rise firstly as the amplitude increases. Then the lift force starts to drop and the damping effect becomes bigger so that the main traits of VIV such as selfexcitation and self-limitation can be effectively captured during VIV response.

\section{Effects of top-end vessel sway on riser's VIV}

By combining the presented hydrodynamic model with the structure model, we can run the dynamic response calculation of the integrated system (shown in Fig. 1) by using FEM code (Chen et al., 2012). In order to explore the impacts of top-end vessel sway on riser's VIV, we present the riser response displacements and vibration wave propagations along riser length while the sway amplitudes or frequencies change.

The structural parameters of the riser are as follows: the outer and inner diameters are respectively $D=0.500 \mathrm{~m}$ and $d=0.445 \mathrm{~m}$. The riser length is $500 \mathrm{~m}$, and the material density is $\rho_{s}=7.8 \times 10^{3} \mathrm{~kg} / \mathrm{m}^{3}$. The bending stiffness is $E I=3.8 \times 10^{7} \mathrm{~N} \mathrm{~m}^{2}$ and the structural damping ratio is 0.03 . The top tension is $T=6.8 \times 10^{5} \mathrm{~N}$ and the flow velocity is $U=1.0 \mathrm{~m} / \mathrm{s}$.

\subsection{Comparison with the case without top-end motion}

To examine what will happen when top-end vessel is swaying, we compared the dynamic responses of the risers between two cases, i.e. Case 1: the riser is suffering only VIV, and Case 2: the riser is suffering both VIV and vessel sway.

Given that the riser dynamic response becomes larger when the frequency of vessel sway is approaching the natural frequencies of the riser, we chose the sway frequency that is consistent with the natural frequency of the rise. Selected displacements are presented in Fig. 4 where the top-end is swaying at the 6th natural frequency of the riser. It is shown that the maximum RMS displacement (see Fig. 4b) of the riser experiencing both top-end sway and VIV is around 2.60, while the maximum RMS displacement (see Fig. 4a) is just 0.48 for the riser experiencing VIV alone. So, we may say that top-end sway could introduce a larger riser response than the case without top-end motion.

Moreover, if comparing the displacement amplitude of the riser's top-end with the amplitudes of the points at other positions along riser length (see Fig. 4b), we note that the RMS displacements of the six peaks range from 2.30 to 2.60 , while the displacement at the topend $(z / L=1.0)$ is just 0.75 . This demonstrates that the displacement amplitudes along riser length get larger than that of the top-end. In other words, the vibration amplitude becomes larger, or is amplified, as the vibration propagates from top-end to bottom end along riser length. We call this phenomenon as response amplification.
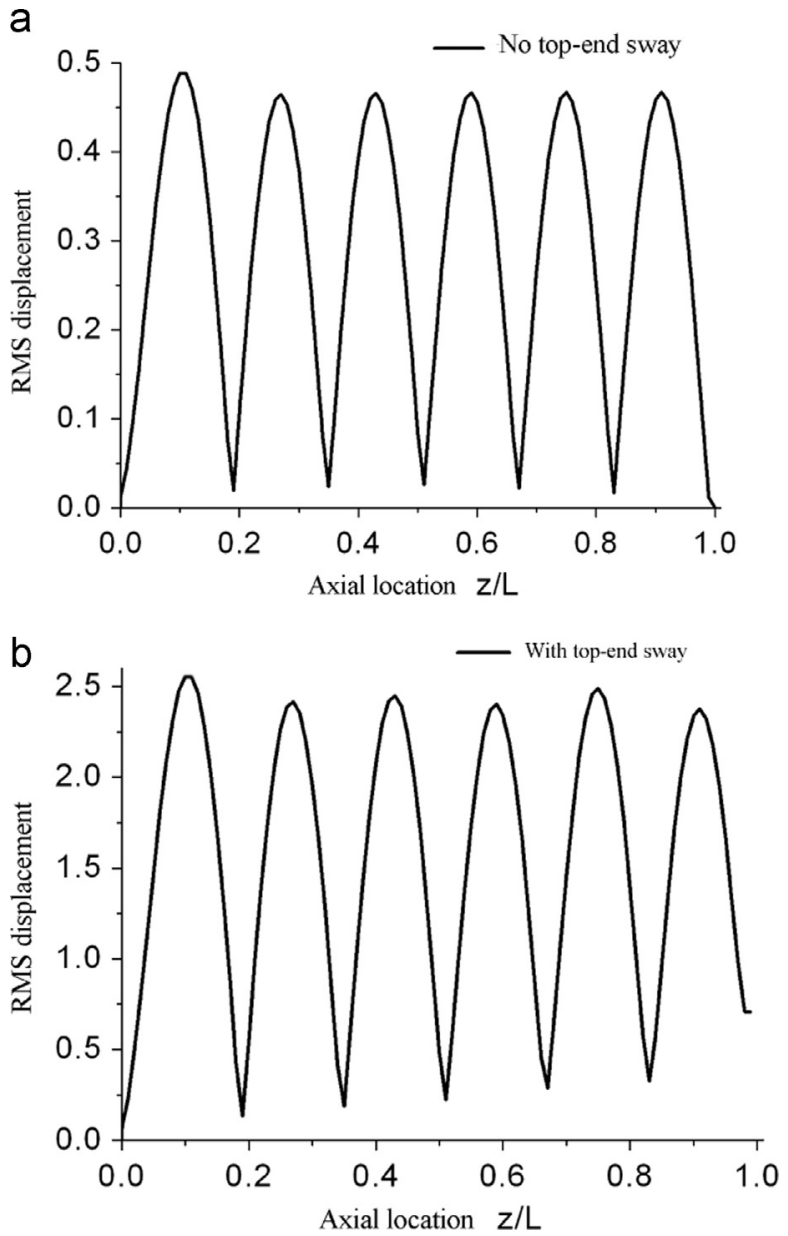

Fig. 4. Comparison between responses with and without top-end motion: (a) riser response without top end motion and (b) riser response with top end motion.

The amplification may be attributed to the moving boundary condition at riser's top-end, of which the mechanism will be further discussed in Section 3.4.

\subsection{Influences of sway frequencies}

The riser dynamic responses are simulated at different frequencies of top-end sway while the top-end is swaying with a $10 \mathrm{~m}$-amplitude. Both the sway cycle and the vortex-induced lift force cycle are same with the riser natural frequencies ranging from the 1st to the 24th modes. Selected dynamic response displacements are presented in Fig. 5. Generally speaking, the displacements decrease as natural frequencies increase. Or, the amplification factor (ratio of maximum dispacment to top-end amplitude) drops with the increase of modal order number. Because, as frequency climbs, the modal order number rises too. As we know, the lower modes usually contribute much more to the dynamic response of practical structure than higher modes. Or, higher modes contribute little or, or even, zero to overall dynamic reponse due to their higher frequencies along with faster damping.

It is also noted that the modal responses of lower-order modes are mostly dominated by standing wave, while the traveling wave can be seen obviously during the responses of higher-order modes, see Fig. $5 d-f$ where there is no longer any exact node. This is mainly because the damping of the modes with higher order number becomes larger, and the modal dynamic response declines faster. Thus, the riser vibration introduced by top-end fluctuation may decline rapidly into a pretty little, even zero, value before it reaches the riser bottom end, and then, to reflect backward. If there is just a pretty small, or even zero, reflected wave to meet with the approaching wave, it is harder to 

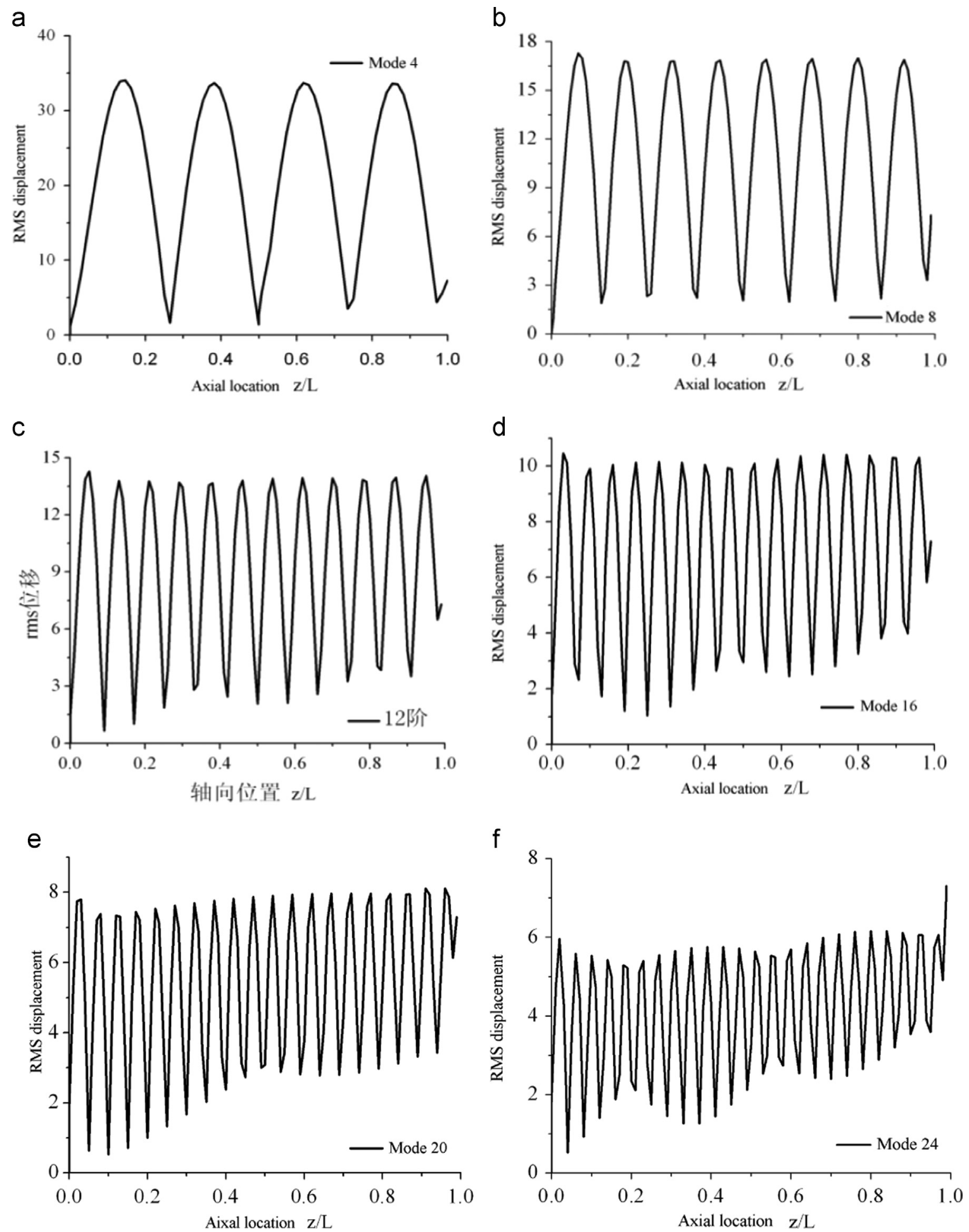

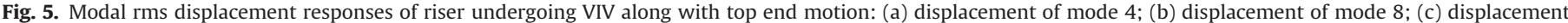
of mode 12; (d) displacement of mode 18; (e) displacement of mode 22 and (f) displacement of mode 24.

form a standing wave for the modes with higher order number. In that case, the vibration wave would be characterized as a traveling wave.

\subsection{Influences of sway amplitudes}

Besides sway frequency, sway amplitude is another important factor which may influence dynamic response of the system. In fact, if normalized by the riser diameter $D$, the sway amplitude $B$ can be redefined as the $K C$ number, $K C=2 \pi B / D$, which implies the relative motion between the top-end and the flow field. Then, the riser responses are simulated for the cases of the sway amplitude ranging from $B / D=1$ to 10 . Selected RMS displacements are shown in Fig. 6 where the sway frequency is $\omega_{6}$, i.e. the 6 th natural frequency of the riser.

As shown in Fig. 6, the riser's displacement gets larger as the sway amplitude rises, e.g. the maximum RMS displacement increases from 2.6 to 16.5 as the sway amplitude increases form $B / D=1$ to $B / D=10$. However, if we make a plot of the non-dimensional parameters (see Fig. 7), the impact of sway amplitude on riser's dynamic response 


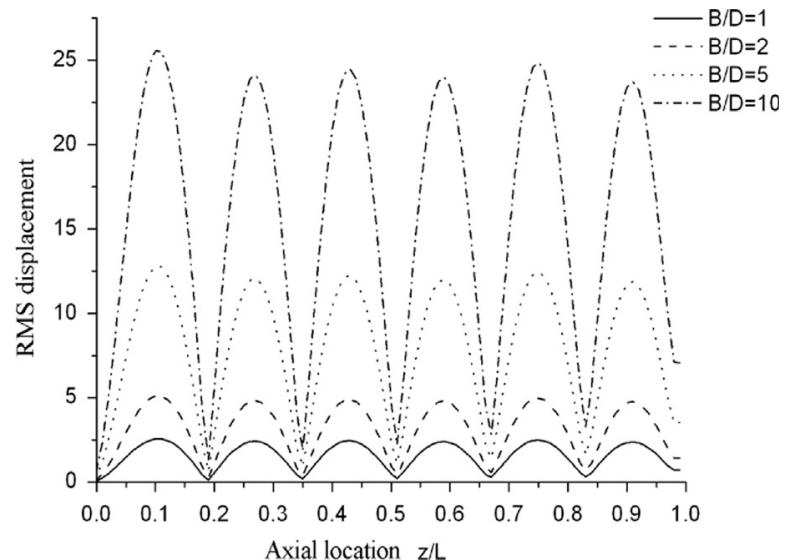

Fig. 6. RMS displacement of riser undergoing different amplitudes of top motion.

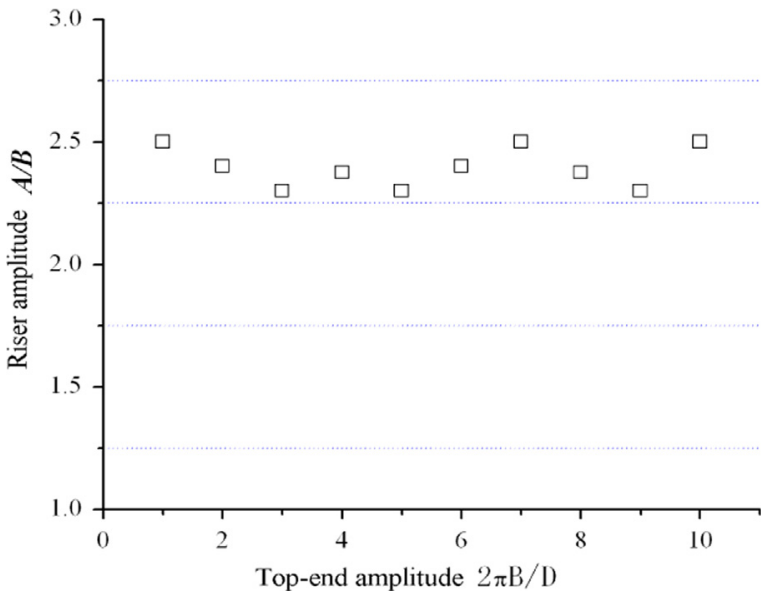

Fig. 7. Effect of KC number on amplification factor.

dramatically changes. In Fig. 7, the displacement is normalized as $A / B$, a ratio of the riser amplitude to the top-end amplitude, and the sway amplitude is normalized as the $K C$ number $2 \pi B / D$. Here we can see that the values of the displacement $A / B$ just slightly fluctuate around 2.5 for all ranges of sway amplitude. Or, the non-dimensional displacement of the riser changes little as the $K C$ number of sway changes. So, we may say there is no profound influence of sway amplitude on riser's amplification factor.

\subsection{Discussion on effect of moving top-end}

To theoretically demonstrate the impacts of moving boundary condition on the riser dynamic response, here we take an Euler beam as example, of which the governing equation can be written as

$E I \frac{\partial^{4} x(z, t)}{\partial z^{4}}-T \frac{\partial^{2} x(z, t)}{\partial z^{2}}+c \frac{\partial x(z, t)}{\partial t}+m \frac{\partial^{2} x(z, t)}{\partial t^{2}}=0$

One end of the beam is fixed, and another end is ongoing a sinusoidal vibration with the frequency $\omega_{0}$ and amplitude $B$. Thus the boundary conditions are

$\left\{\begin{array}{cc}x(L, t)=B e^{-i \omega_{0} t}, & x^{\prime \prime}(L, t)=0 \\ x(0, t)=0, & x^{\prime \prime}(0, t)=0\end{array}\right.$

Assuming a solution, $x=e^{i(k x-\omega t)}$, of Eq. (5), we can get a dispersion equation, by substituting the assumed solution into Eq. (5), as follows:

$E I k^{4}+T_{0} k^{2}-m \omega^{2}-i c \omega=0$ where $k$ is the wave number. By solving Eq. (7), we get the four complex roots, $k= \pm k_{1}, \pm k_{2}$, as

$$
\begin{aligned}
& k_{1}=\sqrt{\frac{1}{2}\left(-\frac{T_{0}}{E I}+\sqrt{\left.\left(\frac{T_{0}}{E I}\right)^{2}+\frac{4\left(m \omega^{2}-i c \omega\right)}{E I}\right)}\right.} \\
& k_{2}=i \sqrt{\frac{1}{2}\left(\frac{T_{0}}{E I}+\sqrt{\left.\left(\frac{T_{0}}{E I}\right)^{2}+\frac{4\left(m \omega^{2}-i c \omega\right)}{E I}\right)}\right.}
\end{aligned}
$$

Then the response displacement can be written as

$x=\alpha_{1} e^{i\left(k_{1} x-\omega t\right)}+\beta_{1} e^{i\left(-k_{1} x-\omega t\right)}+\alpha_{2} e^{i\left(k_{2} x-\omega t\right)}+\beta_{2} e^{i\left(-k_{2} x-\omega t\right)}$
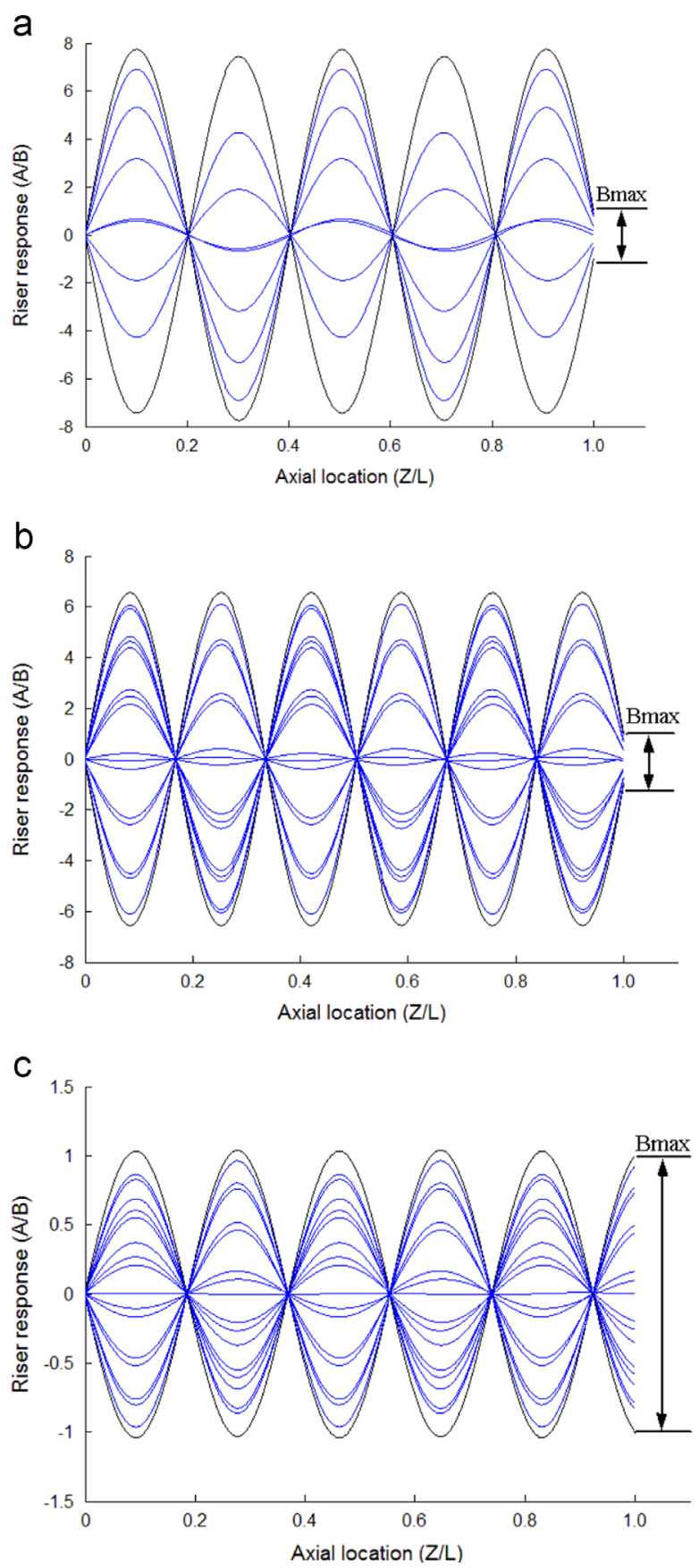

Fig. 8. Trace of the response at different frequencies: (a) modal trace of response at the frequency $\omega_{5}$; (b) modal trace of response at the frequency $\omega_{6}$ and (c) trace of the response at the frequency $1.94 \mathrm{~Hz}$ (not a natural frequency). 
By substituting the boundary conditions, Eq. (6), into Eq. (9), we get an equation group with respect to $\alpha_{1}, \alpha_{2}, \beta_{1}$ and $\beta_{2}$ as follows:

$$
\left[\begin{array}{cccc}
1 & 1 & 1 & 1 \\
-k_{1}^{2} & -k_{1}^{2} & -k_{2}^{2} & -k_{2}^{2} \\
e^{i k_{1} L} & e^{-i k_{1} L} & e^{i k_{2} L} & e^{-i k_{2} L} \\
-k_{1}^{2} e^{i k_{1} L} & -k_{1}^{2} e^{-i k_{1} L} & -k_{2}^{2} e^{i k_{2} L} & -k_{2}^{2} e^{-i k_{2} L}
\end{array}\right]\left\{\begin{array}{c}
\alpha_{1} \\
\beta_{1} \\
\alpha_{2} \\
\beta_{2}
\end{array}\right\}=\left\{\begin{array}{l}
B \\
0 \\
0 \\
0
\end{array}\right\}
$$

Then by solving Eq. (10), the expressions of the coefficients $\alpha_{1}, \alpha_{2}$, $\beta_{1}$ and $\beta_{2}$ can be written as

$$
\begin{array}{ll}
\alpha_{1}=\frac{-B k_{2}^{2} e^{-i k_{1} L}}{-k_{2}^{2} e^{-i k_{1} L}+k_{1}^{2} e^{-i k_{1} L}+k_{2}^{2} e^{i k_{1} L}-k_{1}^{2} e^{i k_{1} L}}, & \alpha_{2}=\frac{B k_{1}^{2} e^{-i k_{2} L}}{-k_{2}^{2} e^{-i k_{2} L}+k_{2}^{2} e^{i k_{2} L}+k_{1}^{2} e^{-i k_{2} L}-k_{1}^{2} e^{i k_{2} L},} \\
\beta_{1}=\frac{B k_{2}^{2} e^{-i k_{1} L}}{-k_{2}^{2} e^{-i k_{1} L}+k_{1}^{2} e^{-i k_{1} L}+k_{2}^{2} e^{i k_{1} L}-k_{1}^{2} e^{i k_{1} L}}, & \beta_{2}=\frac{-B k_{1}^{2} e^{i k_{2} L}}{-k_{2}^{2} e^{-i k_{2} L}+k_{2}^{2} e^{i k_{2} L}+k_{1}^{2} e^{-i k_{2} L}-k_{1}^{2} e^{i k_{2} L}}
\end{array}
$$

With the above solutions, we can carry out dynamic response analysis of a beam with moving boundary condition. Fig. 8 presents the traces of the beam response during a period of time at three different vibrating frequencies, i.e. $\omega_{0}$ is consistent with the beam natural frequencies $\left(\omega_{5}\right.$ and $\left.\omega_{6}\right)$ and a random frequency $\omega_{0}=1.94 \mathrm{~Hz}$. In Fig. 8 the left end of the beam is fixed while the right end is moving sinusoidally with the amplitude of $B=1 \mathrm{~m}$. In Fig. $8 \mathrm{a}$ and $\mathrm{b}$, it is seen that the displacements at the peaks along the beam are obviously larger than the displacements of the right end when the boundary moving frequency is consistent with the beam natural frequency. As mentioned above, we call it response amplification due to moving boundary condition. Moreover, comparing the maximum displacements of Fig. 8a with that of Fig. 8b, we note that the response amplification factor, $A / B$, gets larger when the mode order number is lower, e.g. the value of the amplification factor is 8.0 for mode 5 whereas 6.8 for mode 6 . If the boundary moving frequency is a random value, i.e. not consistent with the beam natural frequency (see Fig. 8c), the response amplification, around 1.1 , may not be as pronounced as that at the natural frequencies.

\section{Conclusions}

A coupled hydrodynamic force approach is developed based on the interaction between fluid and structural dynamics. The dynamic responses of the riser suffering both a floating top-end and VIV are examined by means of finite element numerical simulations. Our results show that the riser displacement becomes several times larger than that of the riser without moving top-end. An interesting phenomenon, that top-end vibration may be amplified as it is propagating from the top to the bottom end along riser length, is observed during the dynamic responses. And, the response amplification gets more pronounced as the number of mode order falls. Based on our numerical simulations, we draw the following conclusions:

(1) Generally speaking, both the displacement and the response amplification of the riser get larger as sway frequencies drop. The dynamics responses of the modes with lower order number are mostly dominated by standing wave, while traveling wave can be observed during the responses of the modes with higher order number.

(2) Riser's displacement gets larger as sway amplitude rises. However, the value of non-dimensional displacement, $A / B$, changes little with the increase of top-end sway amplitude. Or, there is no remarkable impact of sway amplitude on response amplification.

In summary, for an integrated system of a top-end vessel together with submarine riser, the periodical motion of top-end vessel actually introduces a moving boundary condition and, probably, a consequence of response amplification to submarine riser. This response amplification is crucial to dynamic response analysis of practical riser design, because that may directly cause a larger displacement or higher stress-level somewhere along riser length. Large displacement and frequent high stress-level, that are likely to cause fatal damage or to shorten fatigue life of structures, are unwelcome to the safety of deep water platform. In addition, the relative motion of riser to amid fluid field changes the velocity distribution of flow along riser length, that may introduce different vortex shedding modes and consequently new lock-in region. Therefore, a smaller top-end motion may turn out a larger riser motion along with unexpected riser's VIV.

Finally, moving top-end causes a periodical motion of submarine riser, and, consequently, the relative motion between riser and fluid field is no longer steady but unsteady. In that case, a new issue, unsteady VIV which is quite different from steady VIV, may need to be considered. Hopefully, further studies on unsteady VIV, such as new important factors characterizing unsteadiness, lift force model and VIV prediction approach particularly for VIV under unsteady flow conditions, will be addressed, though these issues will be more challenging.

\section{Acknowledgment}

The authors would like to thank the financial support of the National Natural Science Foundation of China (Grant nos. 11232012 and 11372320) for this work.

\section{References}

Bosman, R.L.M., Hooker, J., 1999. Elastic modulus characteristics of polyester mooring ropes. In: Proceedings of the Annual Offshore Technology Conference, pp. 1246-1251.

Chen, W.M., Li, M., Zheng, Z.Q., 2012. Dynamic characteristics and VIV of deepwater riser with axially varying structural properties. Ocean Eng. 42, 7-12.

Chen, X.H., Ding, Y., Zhang, Y., 2006. Coupled dynamic analysis of a mini TLP: comparison with measurements. Ocean Eng. 33, 93-117.

Garrett, D.L., 2005. Coupled analysis of floating production systems. Ocean Eng. 32, $802-816$.

Gopalkrishnan, R., 1993. Vortex Induced Forces on Oscillating Bluff Cylinders (Ph.D. thesis). MIT, Cambridge, MA, USA.

Govardhan, R. Williamson, C.H.K., 2004. Critical mass in vortex-induced vibration of a cylinder. Eur. J. Mech. B/Fluids 23, 17-27.

Gu, J.Y., Lu, H.N., Yang, J.M., 2012. Studies on coupling dynamic response and characteristics mooring system of TLP in stochastic waves. Ocean Eng. 30 (4), 42-48 (in Chinese).

Heurtier, J.M., Buhan, L., Fontaine, E., 2001. Coupled dynamic response of moored FPSO with risers. In: Proceedings of the Eleventh International Offshore and Polar Engineering Conference, pp. 17-22.

Kim, M.H., Arcandra, T., Kim, Y.B., 2001. Variability of spar motion analysis against various design methodologies/parameters. In: Proceedings of the ASME 20th International Conference on Ocean, Offshore and Arctic Engineering, OMAE2001. Rio de Janeiro, Brazil, June.

Lee, H.H., Wang, P.W., 2000. Analytical solution on the surge motion of tension-leg twin platform structural systems. Ocean Eng. 27, 393-415.

Lei, S., Zhang, W.S., Zhao, Y., Yue, Q.J., 2010. Vortex-induced vibration of FPSO riser. Eng. Mech. 27I, 294-298 (in Chinese).

Li, B.B., Ou, J.P., Teng, B., 2010. Fully coupled effects of hull, mooring and risers model in time domain based on an innovative deep draft multi-spar. China Ocean Eng. 24 (2), 219-233.

Lou, M., Dong, W.Y., Wang, T., 2010. Study on dynamic response of marine riser in floating system with pitch and sway motions. China Offshore Platform 25 (4), 14-18 (in Chinese).

Ormberg, H., Fylling, I.J., Larsen, K., Sodahl, N., 1997. Coupled analysis of vessel motions and mooring and riser system dynamics. In: Proceedings of the ASME 16th International Conference on Ocean, Offshore and Arctic Engineering OMAE1997. Yokohama, Japan, April.

Sarpkaya, T., 2004. A critical review of the intrinsic nature of vortex-induced vibration. J. Fluids Struct. Mech. 46, 389-447.

Spanos, P.D., Ghosh, R., Finn, L.D., 2005. Coupled analysis of a spar structure: Monte Carlo and statistical linearization solutions. J. Offshore Mech. Arc. Eng. 127 (1), $11-16$.

Stansberg, C.T., Ormberg, H., Oritsl, O., 2002. Challenges in deep water experiments: hybrid approach. J. Offshore Mech. Arc. Eng. 124, 91-96.

Tahara, A., Kim, M.H., 2003. Hull/mooring/riser coupled dynamic analysis and sensitivity study of a tanker-based FPSO. Appl. Ocean Res. 25, 367-382.

Tahara, A., Kim, M.H., 2008. Coupled-dynamic analysis of floating structures with polyester mooring lines. Ocean Eng. 35 (17-18), 1676-1685. 
Vandiver, J.K., 2002. A universal reduced damping parameter for prediction of vortex-induced vibration. In: Proceedings of the ASME 21st International Conference on Ocean, Offshore and Arctic Engineering OMAE2002. Oslo, Norway, June.

Wang, D.Y., Ling, G.C., 1998. Vortex-induced nonlinear vibration of TLP tethers under circumstances of platform oscillation. Acta Oceanol. Sin. 20 (5), 119-128 (in Chinese).
Wichers, J.E.W., Voogt, H.J., Roelofs, H.W., Driessen, P.C.M., 2001. DeepStar-CTR 4401 Benchmark Model Test. Technical Report No. 16417-1-OB. MARIN, Netherlands. Xu, W.H., Zeng, X.H., Wu, Y.X., 2009. Coupled dynamic response of the tension leg platform and tendon in deep water. J. Vib. Shock 28 (2), 145-150 (in Chinese). 